\title{
Effect on rheological properties with different carboxyl density of polycarboxylate superplasticizer
}

\author{
Chengfei Fu ${ }^{1, \text { a }}$, Hui Zhou ${ }^{1}$, Minghua Yuan ${ }^{1}$, Xing $\mathrm{Li}^{1}$, Long Xiong ${ }^{1}$ \\ ${ }^{1}$ China Construction Ready Mixed Concrete Co. Ltd, Wuhan 430205, China \\ afuchengfei0816@163.com
}

Keywords: polycarboxylate superplasticizer, carboxyl density, molecular structure, rheological properties

\begin{abstract}
Under the condition of unchangeable reaction time, the dosage of initiator and chain transfer agent, the molar volume of unsaturated double bond, we synthesized a series of polycarboxylate superplasticizer via free radical polymerization. These polycarboxylate superplasticizer had a similar structure except carboxyl density. They were investigated by Gel Permeation Chromatography (GPC) and High Performance Liquid Chromatography (HPLC). On this basis the properties of this PCE was evaluated through methods of rheological test. It showed that the dispersility was better as we increased the carboxyl density, while the risk of bleeding was increased.
\end{abstract}

\section{Introduction}

Rheological properties of cement paste is an important method to evaluate the dispersibility of polycarboxylate superplasticizers[1-4]. We can't explain the inherent relationship between rheological property and molecular of polycarboxylate superplasticizers simply via the fluidity of cement paste. In recent years, researchers use rheological methods to get rheological parameters of cement past. They evaluate the rheological property through shear and yield stress, plastic viscosity, apparent viscosity, thixotropism, etc. These can provide strong evidence for the dispersibility of polycarboxylate superplasticizers[5-7].

Research shows that the rheological properties of fresh concrete generally conform to the rheological model of Bingham or Herschel-Bulkley [8-9].

Table 1. Different formulas of the model

$$
\begin{array}{ll}
\text { Bingham } & \tau=\tau_{0}+\mu \cdot \gamma \\
\text { Herschel- Bulkley } & \tau=\tau_{0}+\mu \cdot \gamma^{n}
\end{array}
$$

( $\tau=$ shear stress, $\tau_{0}=$ shear and yield stress, $\mu=$ plastic viscosity, $\gamma=$ shear rate, $\mathrm{n}=$ shear index)

Bingham fluid model is only applicable to plastic fluid while Herschel-Bulkley is suitable for different fluid because of different $\mathrm{n}$. Considering the rheological test in this work is tested in the same dosage of PCE and water cement ratio, the rheological properties of cement paste may have big difference as different dispersibility of polycarboxylate superplasticizers. Therefore, this work adopts Herschel-Bulkley as basic medol to fit flow curve.

\section{Experimental}

\subsection{Chemicals and Equipment.}

Prenyl alcohol ethoxylates (TPEG, Shanghai Taijie Chemical Co. LTD), Acrylic acid (AA,Wuhan Zhonghua Yongye Chemical Co. LTD), Ammonium persulfate (Aps, Shanghai degussa-aj chemical Co. LTD), Chain transfer agent (MAS, Changzhou Yurong Chemical Co.LTD), HuaXin cement,P.O42.5. 


\subsection{GPC Analysis method}

Molecular weight of PCE was measured by Gel Permeation Chromatography (GPC, Waters 150C, USA), using the solution of methanol and buffer solution $(1 / 2, v / v)$ as the eluent. (Column, PL aquagel, mix/m, 7.8*300 mm, Agilent1100, Agilent Company, USA). The buffer solution was $\mathrm{Na}_{2} \mathrm{~B}_{4} \mathrm{O}_{7} / \mathrm{KCl}$ aqueous solution $(\mathrm{pH}=8)$. Narrow polyethylene glycol (PEG) was used as calibration standards. The GPC detector included the refractive index.

\subsection{Synthesis of PCE}

Put a flask within water and TPEG into water-bath heater, and stirried until the end of experiment; wait until TPEG dissolved, then added the APS at certain temperature, and dripped the AA and TGA solution for 3-3.5h; keep heating for $1 \mathrm{~h}$, then decreased temperature; added the sodium hydroxide and water, and adjusted $\mathrm{pH}$ of the new product about 7.

\subsection{Test of slump flow and rheological properties}

Testing of the fluidity of cement pastes was under the national standard GB 8077-2000, the dosage of the PCE was $0.15 \%$ (by percent Weight based on solids cement). The rheological test adopted Paddle method, instrument is R/S-SST made in US Brookfielf company, test system adopted V40-20-3to1 rotor, RheoV2.8 was used to process the data.

\section{Results and Discussion}

\subsection{Relation between molecular structure and carboxyl density}

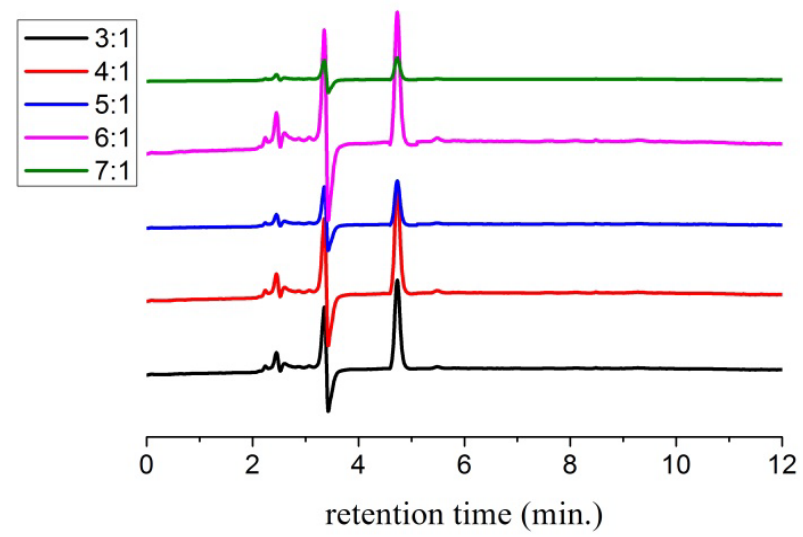

Fig.1 Effect on monomer conversion with different carboxyl density

Table 2. Effect on molecular weight with different carboxyl density

\begin{tabular}{ccccc}
\hline Carboxylic acid/Polyether & Mw & Mn & DPI. & Mp \\
\hline $3: 1$ & 9669.4 & 5816.6 & 1.7 & 3784.8 \\
$4: 1$ & 10986.1 & 6220.9 & 1.8 & 5040.4 \\
$5: 1$ & 11910.2 & 6377.4 & 1.9 & 5040.4 \\
$6: 1$ & 11851.2 & 6360.2 & 1.9 & 5040.4 \\
$7: 1$ & 13930.1 & 6798.8 & 2.0 & 5040.4 \\
\hline
\end{tabular}

Table 2 showed polycarboxylate superplasticizers with different carboxyl density had similar molecular weight, it explained that we gained similar structure of polycarboxylate superplasticizers via this method. 


\subsection{The effect on rheological properties with different carboxyl density}

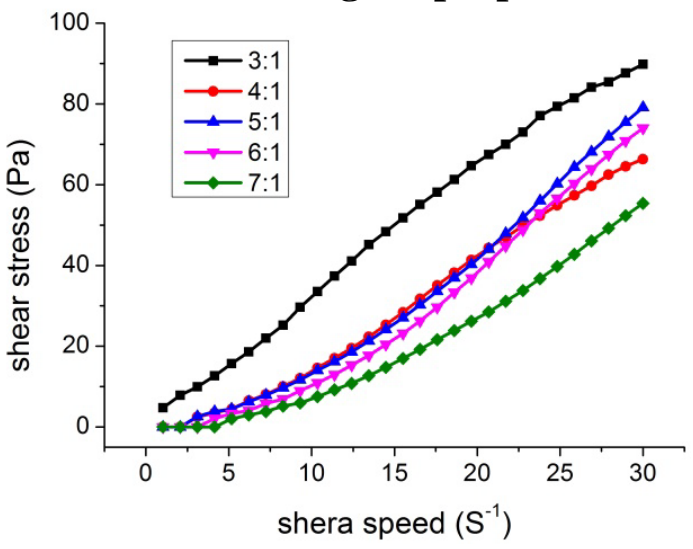

Fig.2 Relation between shear rate and

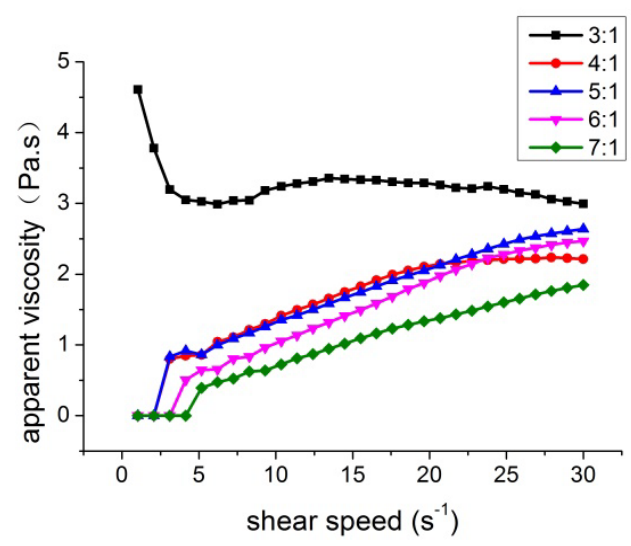

Fig.3 Relation between shear rate and apparent viscosity

Fig.2 showed that shear stress decreased while shear rate increased when carboxyl density below 4:1, cement paste acted as plastic fluid. Stress increased while shear rate increased when carboxyl density exceeded $4: 1$, cement paste acted out dilatancy. When carboxyl density equal to $4: 1$, cement paste acted as shear dilatant fluid if shear rate was low, while in the opposite cement paste acted as plastic fluid.

Fig.3 showed that apparent viscosity increased while shear rate increased when carboxyl density below 4:1, cement paste obviously acted out dilatancy. Stress increased while shear rate increased when carboxyl density exceeded 4:1, cement paste acted out dilatancy. When carboxyl density equaled to 4:1, cement paste acted as shear dilatant fluid if shear rate was low, while in the opposite cement paste acted as plastic fluid. When carboxyl density equaled to 4:1, apparent viscosity of cement paste increased as shear rate increased, then stay stable. When carboxyl density was less than 4:1, apparent viscosity of cement paste decreased as shear rate increased and acted out plastic fluid.

We named it shear yield stress which needed critical stress of cement paste from still to flow, it was critical factor if cement paste could flow. Rheological property was better while shear yield stress got smaller. Plastic viscosity was related to water conservation of cement paste, it was easily bleeding when plastic viscosity was small.

Table 3. Effect on rheological property and medol with different carboxyl density

\begin{tabular}{cccccc}
\hline $\begin{array}{c}\text { Carboxylic } \\
\text { acid/Polyether }\end{array}$ & Fitting Equation & $\begin{array}{c}\text { Correlation } \\
\text { Coefficient }\end{array}$ & $\begin{array}{c}\text { Residual } \\
\text { Standard } \\
\text { Deviation }\end{array}$ & $\begin{array}{c}\text { Shear Yield } \\
\text { Stress (Pa) }\end{array}$ & $\begin{array}{c}\text { Plastic } \\
\text { Viscosity } \\
\text { (Pa.s) }\end{array}$ \\
\hline $3: 1$ & $\tau=0+3.7685 \gamma^{0.9442}$ & 0.99597 & 1.77 & 0 & 3.7685 \\
$4: 1$ & $\tau=0+0.6411 \gamma^{1.3802}$ & 0.99378 & 1.71 & 0 & 0.6411 \\
$5: 1$ & $\tau=0.0181+0.3079 \gamma^{1.6372}$ & 0.99946 & 0.58 & 0.0181 & 0.3079 \\
$6: 1$ & $\tau=0+0.1866 \gamma^{1.7708}$ & 0.99773 & 1.14 & 0 & 0.1866 \\
$7: 1$ & $\tau=0+0.1762 \gamma^{1.5408}$ & 0.99825 & 1.12 & 0 & 0.1762 \\
\hline
\end{tabular}

Table 3 showed that when molecular weight stayed stable, plastic viscosity got smaller while carboxyl density increased. Shear yield stress of cement paste almost reached 0 when carboxyl density exceeded 2:1. Under the same amount of PCE, the dispersion of cement paste got better as carboxyl density increased, meanwhile the risk of bleeding was increased. 


\section{Conclusion}

Under the condition of unchangeable reaction time, the dosage of initiator and chain transfer agent, the molar volume of unsaturated double bond, we synthesized a series of polycarboxylate superplasticizer via free radical polymerization. These polycarboxylate superplasticizer had a similar structure except carboxyl density. They were investigated by Gel Permeation Chromatography (GPC) and High Performance Liquid Chromatography (HPLC). On this basis the properties of this PCE was evaluated through methods of rheological test. It showed that the dispersility was better as we increased th carboxyl density, while the risk of bleeding was increased.

\section{References}

[1] Roussel N, Ovarlez G, Garrault S, et al. The Origins of Thixotropy of Fresh Cement Pastes[J]. Cement and Concrete Research, 2012, 42(1): 148-157.

[2] Yamada K. Basics of Analytical Methods Used for the Investigation of Interaction Mechanism between Cements and Superplasticizers[J]. Cement and Concrete Research, 2011, 41(7): 793-798.

[3] Zhang M, Sisomphon K, Ng T S, et al. Effect of Superplasticizers on Workability Retention and Initial Setting Time of Cement Pastes[J]. Construction and Building Materials, 2010, 24(9): 1700-1707.

[4] Basim A. Rheology of Sodium and Calcium Bentonite-water Dispersions: Effect of Electrolytes and Aging Time[J]. International Journal of Mineral Processing, 2011, 98(3-4): 208-213.

[5] Senff L, Hotza D, Repette W L. Rhelogycal Behaviour of Cement Pastes with Addition of Silica Fume, Nanosilica and Polycarboxilic Dispersant[J]. Revista Matéria, 2010, 15(1): 12-20.

[6] Petit J, Wirquin E, Helnan-Moussa B. Effect of W/C and Superplasticizer Type on Rheological Parameters of SCC Repair Mortar for Gravitational or Light Pressure Injection[J]. Cement and Concrete Composites, 2011, 33(10): 1050-1056.

[7] Kaci A, Chaouche M, Andréani P. Influence of Bentonite Clay on the Rheological Behaviour of Fresh Mortars[J]. Cement and Concrete Research, 2011, 41(4): 373-379.

[8] Bentz D P, Ferraris C F. Rheology and Setting of High Volume Fly Ash Mixtures[J]. Cement and Concrete Composites, 2010, 32(4): 265-270.

[9] Libre N A, Khoshnazar R, Shekarchi M. Relationship Between Fluidity and Stability of Self-consolidating Mortar Incorporating Chemical and Mineral Admixtures[J]. Construction and Building Materials, 2010, 24(7): 1262-1271. 